\title{
Organotypic 3D models and tissue microarrays as means for identifying HSF1 as a prognostic marker for prostate cancer survival after radical prostatectomy
}

\author{
Johanna K Björk and Malin Åkerfelt* \\ Institute of Biomedicine, University of Turku, Turku, Finland
}

Prostate cancer is one of the most commonly diagnosed male cancers in Western countries [1]. The clinical course is, however, highly variable: at one end of the spectrum are primary prostate cancer that remains indolent throughout the lifetime of the patient, and in the other end are cancers that progress into castration-resistance and rapidly metastasize. At this stage there are no curative treatments and the metastasizing tumor phenotype is lethal. Gleason score and grade group are the most commonly used tools to evaluate prognosis, however, there is a critical need for additional information that allows precise stratification of patients into distinct prognostic groups. For example, using radical prostatectomy the prostate gland and tissues surrounding it, usually including the seminal vesicles and nearby lymph nodes, are removed. It is a common treatment option for the largest part of the spectrum of patients, however, without precise predictions on the progress of the disease this inevitably leads to unnecessary surgeries. In addition, decision-making on what the most beneficial treatment option is for a given person after radical prostatectomy, is hampered by a lack of reliable biomarkers.

Heat shock factor 1 (HSF1) is a ubiquitously expressed transcription factor initially known for its role as a regulator of the heat shock response, a well conserved protective mechanism activated upon protein damaging stress $[2,3]$. The function of HSF1 has since been shown to include regulation of target genes in various cellular stress response pathways and beyond, such as apoptosis, chromatin remodeling, cell growth and proliferation and aging [4]. However, the beneficial prosurvival role of HSF1 seems exploited by cancer cells, which inevitably suffer from proteotoxic stress raised from e.g. genomic instability or oxidative stress. Using HSF1 to enhance proteomic stability malignant transformation is empowered, resulting in also a pro-oncogenic role for HSF1 [5]. In accordance, enhanced expression and activity of HSF1 has been detected in numerous cancer types [5-8]. Notably, extensive HSF1 target gene profiling in cancer cell lines and types has revealed a cancer-specific transcriptional program distinct from cells exposed to heat shock and, by its magnitude, reshaping the cellular physiology of cancer cells to cope with chronic proteotoxic stress [9]. Clinical relevance for HSF1 has been demonstrated in breast, lung, endometrial, and hepatocellular carcinoma, where high expression correlates with poor prognosis [9-14].

In prostate cancer, initial reports showed that HSF1 expression levels are upregulated in aggressive tumor cell lines, e.g. PC3 cells [15,16]. In the same cell line, it was demonstrated that HSF1 influences cell cycle behavior, progression through mitosis, and promotes development of the aneuploidy state [17]. HSF1 was also found to be important for induction of arylamine $\mathrm{N}$-acetyltransferase (NAT1) in prostate cancer cells and is required for androgen activation of the NAT1 promoter [18]. Based on the implications of HSF1 in prostate cancer cell lines presented, we asked whether HSF2, another HSF family member, also would play a role in prostate cancer. Gene expression profiling together with functional studies revealed that HSF2 functions as a tumor suppressor in prostate cancer invasion [19]. Interestingly, the role for HSF1 in prostate cancer seemed opposite to that of HSF2. In the study, we took advantage of biologically relevant preclinical models, i.e. 3D organoids, which closely recapitulate cancer histology observed in prostate cancer patients. HSF1 was silence in the organoids using siRNA [19]. Prostate cancer-derived PC3 cells were seeded into lamininrich extracellular matrix, Matrigel. Matrigel promotes organotypic acinar structures, denoting organoids that display physiologically relevant cell-cell and cell-matrix interactions, epithelial polarization and differentiation. PC3 cells constitute an experimental model for castration-resistant prostate cancer and invasion, as they transiently differentiate into hollow organoids, later spontaneously de-differentiate, to finally form invasive stellate structures. The cells were transfected with siRNAs (control or HSF1), transferred into the Matrigel, and organoid morphology was monitored using live-cell imaging for eight days (Figure 1, left panel). At the endpoint, the organoid morphology was imaged by confocal microscopy. Automated image data analysis enabled quantification of morphometric features such as organoid size (area), epithelial differentiation (roundness) and invasion (appendages) [19-23]. This approach revealed that silencing of HSF1 perturbed acinar differentiation, increased the frequency of cell death, and blocked invasion of prostate cancer cells (Figure 1, right panel, [19]). Notably, silencing of HSF1 did not markedly affect cell proliferation or morphology in standard 2D cell cultures on plastic, demonstrating the importance of choosing biologically relevant preclinical models for functional studies. Further accentuating this aspect is the fact that comparable results to those obtained using 3D cancer models were obtained when prostate cancer cells were grown on the chorioallantoic membrane of chicken embryos (CAM model). In this model, silencing of HSF1 resulted in loss of tumor growth and less invasive behavior [19].

Elevated mRNA and/or protein levels have previously been detected in prostate cancer cell lines and tumors [9,15,19]. To

*Correspondence to: Malin Åkerfelt, Institute of Biomedicine, University of Turku, 20520 Turku, Finland, Tel: +35-840-525-7891; Fax: +35-823-337-000; E-mail: malin.akerfelt@utu.fi

Received: October 18, 2018; Accepted: October 29, 2018; Published: October 31,2018 
Björk JK (2018) Organotypic 3D models and tissue microarrays as means for identifying HSF1 as a prognostic marker for prostate cancer survival after radical prostatectomy
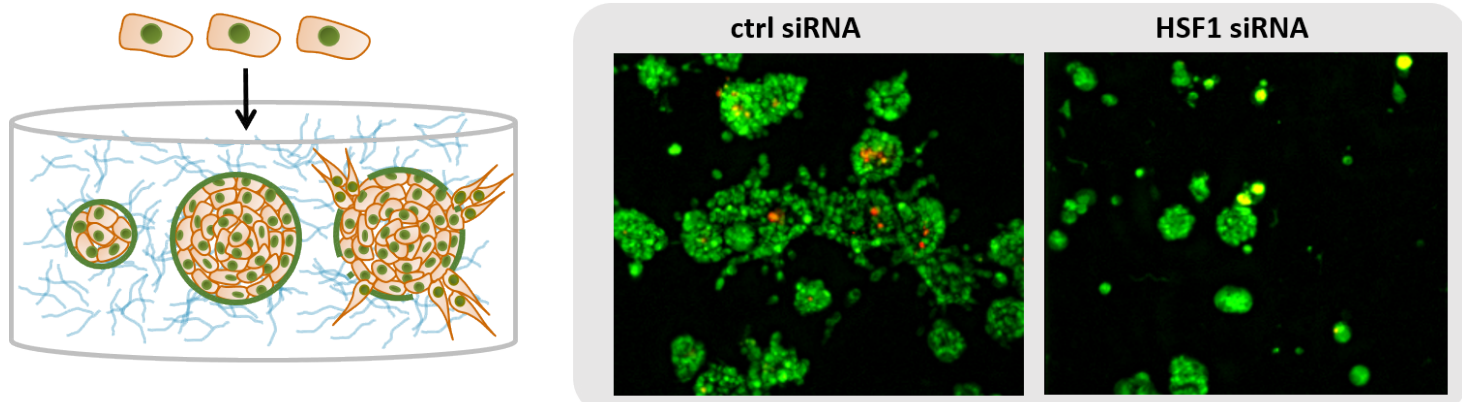

Figure 1. Live cell imagining of organotypic 3D culture models of prostate cancer cell lines was used for identification of HSF1 as a promoter of prostate cancer [19]. A schematic drawing illustrating the organotypic 3D cultures (left), which were labelled with live cell dyes to detect morphological changes in the organoids upon HSF1 silencing (right).
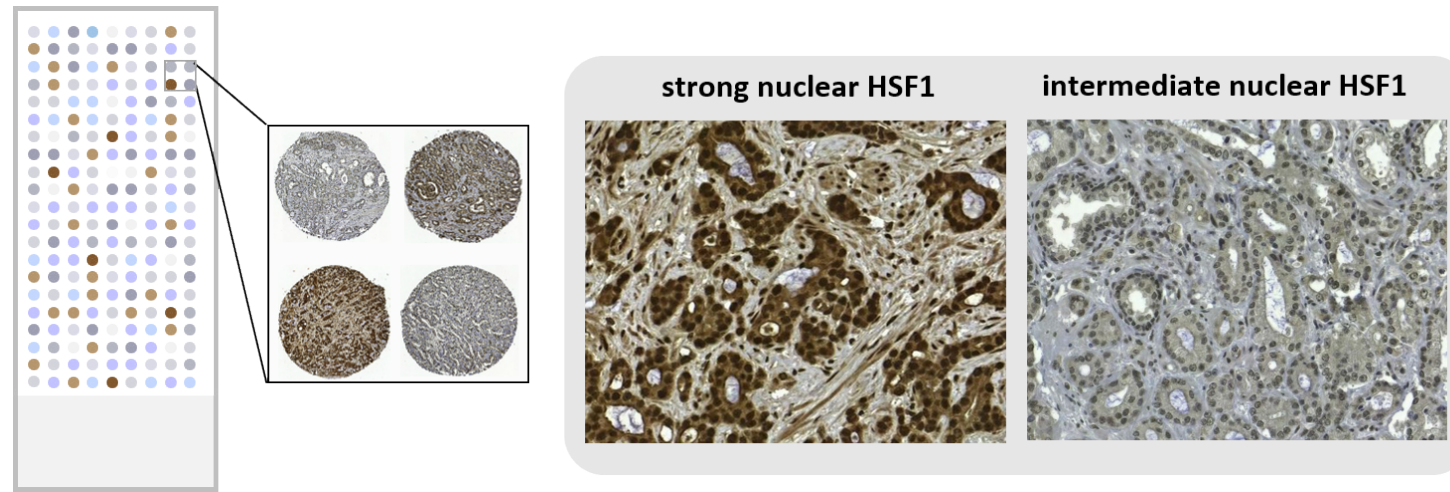

Figure 2. Tissue microarrays from patient samples of primary prostate cancer were used for identifying nuclear HSF1 as a prognostic marker after radical prostatectomy. A schematic drawing illustrating a tissue microarray (left), which was stained with HSF1 antibody to detect differences in expression patterns (right). The stainings were adapted from [24].

focus on the clinical relevance of HSF1 in prostate cancer we asked whether HSF1 activation, i.e. elevated protein levels, could be used as a predictive marker in patients with prostate cancer [24]. Tissue microarrays (TMA) [25] from a comprehensive radical prostatectomy cohort of 478 patients and with extensive follow-up time (15.7 years), to enable clinically relevant endpoint analyses, were utilized (Figure 2). Immunohistological stainings revealed that increased HSF1 expression corresponds to advancement of prostate cancer. Strong nuclear HSF1 staining was associated with shorter secondary therapy-free survival, and in multivariate Cox analysis, taking established clinical markers into account, nuclear HSF1 remained an independent predictor of secondary therapy. Importantly, strong nuclear HSF1 expression increased the likelihood to die from prostate cancer. Using the clinical Cancer of the Prostate Risk Assessment post-Surgical (CAPRA-S) score in multivariable analysis combined with multiple imputation, nuclear HSF1 remained a predictive factor of shortened disease-specific survival. The results indicate that HSF1 could be used as a novel prognostic marker after radical prostatectomy. Limitations of the study include its retrospective nature. Furthermore, even if the size and follow-up time of the patient cohort used were considerable, the relatively low count of lethal events reduced the statistical power in subgroup and multivariate models. For validation, immunohistological staining was performed on an independent TMA cohort comprising of regionally advanced to distant metastatic samples. This confirmed the correlation between high HSF1 levels and advanced and aggressive disease.

Taken together, results are accumulating from various cancer forms, demonstrating activation and high expression of HSF1 when comparing normal and malignant tissue, as well as an association with poor disease outcome [5]. In prostate cancer, we have demonstrated the significance of HSF1 for growth, progression and invasion of the cancer [19] and as a predictor of poor disease-specific survival [24]. Radical prostatectomy constitutes a common treatment for localized prostate cancer. However, also post-surgery, outcome predictions and choice of secondary treatment options, i.e. locoregional or systemic, remain challenging, in particular for intermediate and high-risk patients. Our results indicate the HSF 1 could be used in clinical practice as a prognostic marker after radical prostatectomy. As a next step, prospective studies on extensive, independent cohorts would be informative to elucidate the usefulness. It would also be interesting to pinpoint at which stage of the disease progression HSF1 would be most optimally used as a marker. If, for example, HSF1 status was surveyed in biopsies this could potentially reduce unnecessary radical prostatectomies and facilitate earlier treatment. Another aspect urging attention for future work is the potential of HSF1 as a therapeutic target in prostate cancer. Our studies presented here open up for compound screening targeting HSF1 in organotypic 3D models. A novel traizole nucleoside analogue was recently discovered to exhibit potent anticancer activity via downregulation of HSF1, along with the inhibition of androgen receptor expression and transactivation in prostate cancer cells [26]. Several other small molecule inhibitors of HSF1 have been identified $[5,27]$, and could be tested against prostate cancer. Thus, further studies are warranted to unravel the whole potential of HSF1 as a biomarker and therapeutic target.

\section{References}

1. Ferlay J, Soerjomataram I, Dikshit R, Eser S, Mathers C, et al. (2015) Cancer incidence and mortality worldwide: sources, methods and major patterns in GLOBOCAN 2012. Int J Cancer 136: E359-386. [Crossref] 
Björk JK (2018) Organotypic 3D models and tissue microarrays as means for identifying HSF1 as a prognostic marker for prostate cancer survival after radical prostatectomy

2. Åkerfelt M, Morimoto RI, Sistonen L (2010) Heat shock factors: integrators of cell stress, development and lifespan. Nat Rev Mol Cell Biol 11: 545-555. [Crossref]

3. Vihervaara A, Sistonen L (2014) HSF1 at a glance. J Cell Sci 127: 261-266. [Crossref]

4. Barna J, Csermely P, Vellai T (2018) Roles of heat shock factor 1 beyond the heat shock response. Cell Mol Life Sci 75: 2897-2916. [Crossref]

5. Dai C, Sampson SB (2016) HSF1: Guardian of Proteostasis in Cancer. Trends Cell Biol 26: 17-28. [Crossref]

6. Dai C, Whitesell L, Rogers AB, Lindquist S (2007) Heat shock factor 1 is a powerful multifaceted modifier of carcinogenesis. Cell 130: 1005-1018. [Crossref]

7. Jin X, Moskophidis D, Mivechi NF (2011) Heat shock transcription factor 1 is a key determinant of HCC development by regulating hepatic steatosis and metabolic syndrome. Cell Metab 14: 91-103. [Crossref]

8. Scott KL, Nogueira C, Heffernan TP, van Doorn R, Dhakal S, et al. (2011) Proinvasion metastasis drivers in early-stage melanoma are oncogenes. Cancer Cell 20: 92-103. [Crossref]

9. Mendillo ML, Santagata S, Koeva M, Bell GW, Hu R, et al. (2012) HSF1 drives a transcriptional program distinct from heat shock to support highly malignant human cancers. Cell 150: 549-562. [Crossref]

10. Santagata S, Hu R, Lin NU, Mendillo ML, Collins LC, et al. (2011) High levels of nuclear heat-shock factor 1 (HSF1) are associated with poor prognosis in breast cancer. Proc Natl Acad Sci U S A 108: 18378-18383. [Crossref]

11. Fang F, Chang R, Yang L (2012) Heat shock factor 1 promotes invasion and metastasis of hepatocellular carcinoma in vitro and in vivo. Cancer 118: 1782-1794. [Crossref]

12. Engerud H, Tangen IL, Berg A, Kusonmano K, Halle MK, et al. (2014) High leve of HSF1 associates with aggressive endometrial carcinoma and suggests potential for HSP90 inhibitors. Br J Cancer 111: 78-84. [Crossref]

13. Scherz-Shouval R, Santagata S, Mendillo ML, Sholl LM, Ben-Aharon I, et al. (2014) The reprogramming of tumor stroma by HSF1 is a potent enabler of malignancy. Cell 158 564-578. [Crossref]

14. Gokmen-Polar Y, Badve S (2016) Upregulation of HSF1 in estrogen receptor positive breast cancer. Oncotarget 7: 84239-45. [Crossref]

15. Hoang AT, Huang J, Rudra-Ganguly N, Zheng J, Powell WC, et al. (2000) A novel association between the human heat shock transcription factor 1 (HSF1) and prostate adenocarcinoma. Am J Pathol 156: 857-864. [Crossref]
16. Tang D, Khaleque MA, Jones EL, Theriault JR, Li C, et al. (2005) Expression of heat shock proteins and heat shock protein messenger ribonucleic acid in human prostate carcinoma in vitro and in tumors in vivo. Cell Stress Chaperones 10: 46-58. [Crossref]

17. Wang Y, Theriault JR, He H, Gong J, Calderwood SK (2004) Expression of a dominant negative heat shock factor-1 construct inhibits aneuploidy in prostate carcinoma cells. $J$ Biol Chem 279: 32651-32659. [Crossref]

18. Butcher NJ, Minchin RF (2010) Arylamine N-acetyltransferase 1 gene regulation by androgens requires a conserved heat shock element for heat shock factor-1. Carcinogenesis 31: 820-826. [Crossref]

19. Björk JK, Åkerfelt M, Joutsen J, et al. (2016) Heat-shock factor 2 is a suppressor of prostate cancer invasion. Oncogene 35: 1770-1784. [Crossref]

20. Härmä V, Virtanen J, Mäkelä R, Happonen A, Mpindi JP, et al. (2010) A comprehensive panel of three-dimensional models for studies of prostate cancer growth, invasion and drug responses. PLoS One 5: e10431. [Crossref]

21. Härmä V, Schukov HP, Happonen A, Ahonen I, Virtanen J, et al. (2014) Quantification of dynamic morphological drug responses in $3 \mathrm{D}$ organotypic cell cultures by automated image analysis. PLoS One 9: e96426. [Crossref]

22. Robinson S, Guyon L, Nevalainen J, Toriseva M, Åkerfelt M, et al. (2015) Segmentation of Image Data from Complex Organotypic 3D Models of Cancer Tissues with Markov Random Fields. PLoS One 10: e0143798. [Crossref]

23. Åkerfelt M, Bayramoglu N, Robinson S, et al. (2015) Automated tracking of tumorstroma morphology in microtissues identifies functional targets within the tumor microenvironment for therapeutic intervention. Oncotarget 6: 30035-30056. [Crossref]

24. Björk JK, Ahonen I, Mirtti T, Erickson A, Rannikko A, et al. (2018) Increased HSF1 expression predicts shorter disease-specific survival of prostate cancer patients following radical prostatectomy. Oncotarget 9: 31200-31213. [Crossref]

25. Kononen J, Bubendorf L, Kallioniemi A, Bärlund M, Schraml P, et al. (1998) Tissue microarrays for high-throughput molecular profiling of tumor specimens. Nat Med 4: 844-847. [Crossref]

26. Xia Y, Wang M, Beraldi E, Cong M, Zoubeidi A, et al. (2015) A Novel Triazole Nucleoside Suppresses Prostate Cancer Cell Growth by Inhibiting Heat Shock Factor 1 and Androgen Receptor. Anticancer Agents Med Chem 15: 1333-1340. [Crossref]

27. Whitesell L, Lindquist S (2009) Inhibiting the transcription factor HSF1 as an anticancer strategy. Expert Opin Ther Targets 13: 469-478. [Crossref]

Copyright: (C2018 Björk JK. This is an open-access article distributed under the terms of the Creative Commons Attribution License, which permits unrestricted use, distribution, and reproduction in any medium, provided the original author and source are credited. 